\title{
Evaluación del índice de área foliar con método indirecto y directo en distintas condiciones ambientales en plantaciones dendroenergéticas de Eucalyptus tereticornis Sm.
}

\author{
Evaluation of leaf area index with indirect and direct method \\ in different environmental conditions in dendroenergetic plantations of \\ Eucalyptus tereticornis Sm.
}

\author{
Juan Carlos Valverde ${ }^{*}$ y Dagoberto Arias'
}

\begin{abstract}
1 Instituto Tecnológico de Costa Rica. Escuela de Ingeniería Forestal. Laboratorio de Ecofisiología Forestal y Aplicaciones Ecosistémicas. Cartago, Costa Rica.

* Autor de correspondencia. jcvalverde@tec.ac.cr.
\end{abstract}

\section{RESUMEN}

El índice de área foliar (IAF) es un parámetro relacionado con múltiples variables fisiológicas y de crecimiento en especies arbóreas. Su evaluación se ha realizado con técnicas directa e indirecta; la primera es de carácter destructivo, pero con un nivel de precisión elevado; la indirecta es de rápido desarrollo y no afecta el crecimiento del individuo, pero tiene menor precisión. En este último grupo destacan los sistemas ópticos, específicamente el LAI-2000 y las fotografías hemisféricas (FDH) que son las que han proporcionado los mejores resultados en múltiples especies de plantas. Este trabajo evaluó el uso del LAI-2000 (IAF, en español) y de la FDH tomadas con un dispositivo móvil, usando una lente de ojo de pez y una aplicación móvil; se compararon sus resultados con los del método destructivo. La valoración se hizo en dos coberturas de nubes y cuatro intervalos de velocidad del viento. Los resultados mostraron que el mejor método indirecto fue el FDH-app con el modelo IAF $=0,88 \cdot \mathrm{IAP}+0,17$ con un $\mathrm{R}^{2}$ de 0,83, seguido por el IAF-2000 y FDH-Lente. En cuanto a la nubosidad, se encontró que se tiende a subestimar entre $8 \%$ y $60 \%$ el IAF en cobertura parcialmente nublada, indistintamente del método indirecto, pero se mantuvo el FDH-app como el mejor método. Finalmente, se encontró que vientos superiores a 5 km/h generan variaciones de hasta $60 \%$ en el IAF, ya que producen una aleatoriedad del movimiento de las hojas y, con ello, cambios en la estructura de la copa; es decir, disminuyen la precisión del IAF.

PALABRAS CLAVE: aplicaciones móviles, árboles, fotografía hemisférica digital, LAI-2000, método directo, método indirecto.

\section{ABSTRACT}

Leaf Area Index (LAI) is a parameter related to multiple physiological variables and growth in tree species. Its evaluation has been done through direct and indirect techniques; the first one is a destructive test with a higher level of precision, while the indirect technique, being one of rapid development, does not affect the growth of the individual but yields results with lesser precision. Among this group, the optical systems stand out, specifically LAI-2000 and the hemispheric digital photography (FDH), which yields the best results obtained in multiple plant species tests. This work evaluated the use of LAI-2000 and FDH taken with a mobile device, using a fisheye lens, a mobile application and comparing their results with the destructive method. Also, the assessment was made in two cloud covers and four wind speed ranges. The results showed that the best indirect method was the FDH-app with the model IAF $=0.88$ IAP +0.17 with an $\mathrm{R}^{2}$ of 0,83 , followed by the IAF-2000 and FDH-Lent. It was found a tendency to underestimate the IAF in partially cloudy coverage between $8 \%$ and $60 \%$, regardless of the indirect method, although the FDH-app was maintained as the best method. Finally, it was found that winds ranging bigger than $5 \mathrm{~km} / \mathrm{h}$ generate variations of up to $60 \%$ in the IAF values due to changes in the structure of the treetop generating randomness of the movement of the leaves that infers in the increase of the IAF variability, therefore, decreasing its accuracy.

KEYWORDS: app, trees, digital hemispheric photography, LAI-2000, direct method, indirect method. 


\section{INTRODUCCIÓN}

El índice de área foliar (IAF) es la relación del área del tejido foliar por unidad horizontal de superficie (Dovey y du Toit, 2005; Nguy-Robertson et al., 2014; Poblete-Echeverría, Fuentes, Ortega-Farias, Gonzalez-Talice y Yuri (2015). El IAF se correlaciona con la fotosíntesis, la transpiración y la respiración que se realiza en la copa del árbol y por tanto con el intercambio de materia y energía que tiene el organismo con el ambiente (Bréda, 2003; Mason, Diepstraten, Pinjuv y Lasserre, 2012). De tal forma que disponer de información del IAF simplificaría la generación de modelos fisiológicos de especies y ecosistemas (Pisek, Ryu y Alikas, 2011; Poblete-Echeverría et al., 2015), facilitando la creación de modelos productivos y de metodologías que optimicen el manejo silvicultural y la evaluación de la recuperación ecológica de un ecosistema en el tiempo (Vyas, Mehta, Dinakaran y Krishnayya, 2010). Sin embargo, la estimación del IAF es complicada ya que debe tomar en cuenta la orientación y distribución aleatoria del follaje y evitar la confusión en el cálculo del área foliar con el área de ramillas, ramas o fuste (Chianucci y Cutini, 2013). Ante la complejidad del cálculo del IAF se han desarrollado técnicas directas e indirectas (Bréda, 2003; Mason et al., 2012). Las técnicas directas consisten en cosechar el individuo o parte de su copa y, a partir de relaciones alométricas entre la masa seca de las hojas y el área foliar específica (AFE), se genera el IAF (Dovey y du Toit, 2005) con la ventaja de que permiten una alta precisión, pero con limitaciones de complejidad, costo y perturbación en el desarrollo del individuo (en su defecto provoca la cosecha del árbol) y en muchos casos se imposibilita disponer de mediciones precisas en el tiempo (Chianucci y Cutini, 2013).

En contraste, las técnicas indirectas generan distorsiones mínimas en los árboles y facilitan el cálculo del IAF a partir del índice de área de la planta (IAP) (Dovey y du Toit, 2005), lo cual permite no solo generar factores de corrección sino aumentar la precisión del cálculo a un costo accesible (Chianucci y Cutini, 2013). Una de las técnicas indirectas de mayor aplicación ha sido la óptica, que se ha basado en los principios de la Ley de Beer Lamberth (Jonckheere, et al., 2004) y permite modelar el comportamiento de la luz que traspasa la cobertura de la copa y se resume en la siguiente ecuación:

$$
P(\theta)=\exp \left(\frac{-G(\theta) \Omega(\theta) I A P}{\cos \theta}\right)
$$

Donde P es la fracción gap de la copa, $G$ es la función de proyección del follaje que está relacionada con el ángulo de distribución foliar, $\Omega$ es el índice de agrupación del follaje a parir del ángulo zenit $\theta$ y IAP es el índice de área de la planta, que incluye tanto el área foliar como las ramas y partes del fuste. A partir de dicho principio se ha desarrollado instrumentación y técnicas especializadas como el Tracing Radiation and Architecture of Canopies (TRAC) (Chen, Plummer, Rich, Gower y Norman, 1997), SUNSCAN Canopy Analysis System (Vojtech Turnbull y Hector, 2007), AccuPAR (Lang, 1986), DEMON (Nackaerts, Coppin, Muys y Hermy, 2000), LAI-2000 Plant Canopy Analyzer (Demanrez, Duthoit, Baret, Weiss y Dedieu, 2008) y Hemispherical Photography (Jonckheere, et al., 2004; Denmarez et al., 2008; Lui, Jin y Qi, 2012). Estos dos últimos son los que mayor éxito han tenido debido a su facilidad de adquisición y adaptabilidad a distintas especies, ecosistemas y condiciones ambientales (Dovey y du Toit, 2005; Demarez et al., 2008; Chianucci y Cutini, 2012).

Por otra parte, es relevante destacar que con el auge tecnológico de los dispositivos móviles con cámaras fotográficas de alta resolución, en conjunto con aplicaciones y gadgets que permiten obtener fotografías hemisféricas de buena calidad, se facilita la utilización de tecnología de bajo costo la cual, en complemento con programas especializados como Winscanopy, CAN-EYE y HemiView, permiten calcular el IAF (Chianucci y Cutini, 2012).

Con el uso de cámaras de dispositivos móviles no solo se pretende ahorrar tiempo y costo en el cálculo del IAF sino también desarrollar evaluaciones en condiciones 
climáticas en las que normalmente se corre el riesgo de obtener valores sesgados de IAF. Aspectos como la nubosidad y el viento inciden directamente en el cálculo del IAF y en muchos casos se tiende a subestimar (Demarez et al., 2008; Pisek et al., 2011; Valverde, Guevara-Bonilla, Arias, Briceño y Esquivel, 2017). Los equipos especializados como el LAI-2000 (LI-COR Biosciences, 2010) presentan su óptimo funcionamiento en sitios con cielo totalmente nublado y sin presencia de viento, pero los cambios en estas condiciones inciden en el aumento de la variabilidad del IAF generado por el aumento en la luminosidad de la copa que genera distorsiones que muchos de los algoritmos no corrigen (Zou et al., 2014).

\section{OBJETIVOS}

El presente trabajo planteó, para plantaciones dendroenergéticas de E. tereticornis, los siguientes objetivos: (1) comparar valores del IAF con el método directo e indirecto, (2) evaluar el efecto de la nubosidad en la estimación del IAF con ambas metodologías y (3) determinar los efectos de la velocidad del viento en la estimación del IAF.

\section{MATERIALES Y MÉTODOS}

\section{Especie y sitio de estudio}

Se evaluaron 22 individuos de E. tereticornis de 26 meses de edad, con un diámetro promedio a $1,3 \mathrm{~m}$ sobre el suelo de $(58,3 \pm 2,6) \mathrm{mm}$ y una altura total promedio de $(7,37 \pm 0,63)$ $\mathrm{m}$. La plantación se estableció con una densidad de siembra de 10000 árb ha-1 y no se le aplicó ningún tipo de manejo silvicultural.

El sitio de estudio se ubicó en Cartago, Costa Rica ( $9^{\circ} 50^{\prime} 57.91 " \mathrm{~N}$; 8354'37.27"O) y se evaluó entre los meses de mayo y septiembre del 2017, a una altitud de 1392 metros, con una temperatura media anual de $24{ }^{\circ} \mathrm{C}$ y una precipitación anual de $2100 \mathrm{~mm}$ dispuesta en siete meses lluviosos (Instituto Meteorológico Nacional [IMN], 2018). Según la clasificación de clima de Köppen-Geiger, el clima del sitio es oceánico subtropical de alta montaña.

\section{Medición indirecta}

Se utilizó el IAP (índice de área de la planta) como parámetro generador del IAF, obteniéndose a partir de dos técnicas: (1) un equipo Plant Canopy Analyzer LICOR 2000 y (2) imágenes fotográficas hemisféricas (obtenidas con una aplicación móvil y una lente tipo ojo de pez). Las mediciones se desarrollaron entre las 06:30 a.m. y las 08:30 a.m. (GMT-6 América Central) tanto en un clima totalmente nublado como parcialmente nublado. De igual manera se evaluó la temperatura, la humedad relativa y la velocidad del viento, utilizando una miniestación climática móvil marca Skymate (Speedtech Instruments) modelo SM19. La luminosidad se evaluó con un luxómetro marca Dr.Meter ${ }^{\circledR}$ modelo LX1330B. En ambas técnicas, la copa del individuo se dividió en 4 segmentos y en cada uno se tomó la información a una distancia de $50 \mathrm{~cm}$ de este.

Las mediciones del Plant Canopy Analyzer se realizaron con una unidad LAI-2000 (LI-COR Biosciences, Inc., 2010), la unidad se colocó a $60 \mathrm{~cm}$ sobre el nivel del suelo y se complementó con una tapa de $90^{\circ}$ para bloquear el operador del equipo y la copa de los árboles cercanos. Con respecto al cálculo del IAP, se omitió el quinto anillo del sensor para evitar mediciones de árboles que no fueran de interés, según recomienda Dovey y du Toit (2005).

En cuanto a las fotografías digitales hemisféricas $(\mathrm{FDH})$ se utilizó un dispositivo móvil marca ASUS ${ }^{\circledR}$ Modelo Zenphone 5 con un cámara de 8MP (resolución de 2024 pixeles $\times 2024$ pixeles, en formato $R G B$ ). A la cámara se le colocó un lente ojo de pez marca Vivitar con un angular de $180^{\circ}$ y cobertura de $35 \mathrm{~mm}$; además se utilizó la aplicación móvil Fisheye Pro versión 1.2 (R22 software) para Android. Todas las fotografías se tomaron niveladas a una altura de $60 \mathrm{~cm}$ sobre nivel del suelo y con la dirección del Norte previamente marcada.

\section{Análisis de FDH}

Las imágenes se procesaron con el software CAN_EYE versión 6.3 .13 (INRA Science \& Impact), previamente, ambas técnicas se calibraron según el método descrito en el 
manual CAN EYE (versión 6.3.6) (Weiss et al., 2004). Se limitó el grado de visión de las imágenes (COI) a $60^{\circ}$ y, con la opción de recorte en CAN_EYE, se eliminaron las copas de los árboles que rodeaban a cada individuo bajo estudio con el fin de prevenir distorsiones y sesgos en el cálculo del IAP. Adicionalmente, se utilizó un ángulo zenital de $2,5^{\circ} \mathrm{y}$ un azimutal de $5^{\circ}$ (Mougin et al., 2014) con un análisis de pixeles de tipo "No mixed pixels" (2 clases); el algoritmo seleccionado fue el LAI-2000 4 anillos, debido a que en pruebas previas fue el que mostró mejores resultados.

\section{Medición directa}

Los árboles evaluados con la medición indirecta del IAF se cosecharon y se contabilizaron todas las hojas de su copa. Posterior a esto, se extrajo una muestra de $25 \%$ de la copa siguiendo la metodología de Mason et al., (2012), se escanearon a color y con una resolución de 900 puntos por pulgada con un escáner Epson ${ }^{\circledR}$ modelo TX-110 y se les calculó el AFE con el software WINFOLIA® versión 2012 pro. A continuación, se secaron las hojas a $65^{\circ} \mathrm{C}$ por 48 horas para determinar el peso seco y con él se aplicó la ecuación 1 para determinar el IAF.

$$
L A I=\frac{\left(\sum A F E \cdot \sum P L\right)}{U S}
$$

Donde:

$\sum \mathrm{AFE}=$ sumatoria del área foliar específica $\left(\mathrm{m}^{2} / \mathrm{kg}\right)$

$\sum \mathrm{PL}=$ sumatoria del peso seco de las hojas $(\mathrm{kg})$

US $=$ unidad de área del suelo (en este caso, $1 \mathrm{~m}^{2}$ ).

\section{Evaluación del efecto de la nubosidad y el viento}

Con respecto a la nubosidad, se evaluó el IAP tanto en un cielo totalmente nublado (TN) como en un cielo parcialmente nublado (PN) (las fotografías presentan al menos 50\% de nubosidad). Para comprobar si el porcentaje de nubosidad era el indicado, se implementó una preclasificación de pixeles con el programa GIMP 2.8 (www.gimp.com), ambas condiciones se valoraron sin la presencia de viento (velocidad del viento inferior a $5 \mathrm{~km} / \mathrm{h}$ ).
La valoración del efecto viento únicamente se realizó con la FDH-app debido a la respetabilidad del proceso y a que la cámara se calibró para fotografías en alta velocidad (en 0,60 segundos), permitiendo obtener en cada ráfaga de viento de 6 a 10 fotografías. Las mediciones se tomaron en los mismos supuestos expuestos en la metodología y bajo los siguientes intervalos de velocidad de viento: i) de 0 $\mathrm{km} / \mathrm{h}$ a $5 \mathrm{~km} / \mathrm{h}$ (VV1), ii) $5 \mathrm{~km} / \mathrm{h}$ a $10 \mathrm{~km} / \mathrm{h}$ (VV2), iii) 10 $\mathrm{km} / \mathrm{h}$ a $15 \mathrm{~km} / \mathrm{h}$ (VV3) y iv) mayores a $15 \mathrm{~km} / \mathrm{h}$ (VV4).

\section{Análisis estadístico}

Con respecto a la relación entre las variables IAF e IAP, se aplicó la metodología de Mason et al. (2012), que consistió en regresiones lineales en las cuales, el IAP fue la variable independiente y el IAF la dependiente. Estas variables se evaluaron bajo el modelo de regresión lineal IAF $=a+$ $b$.IAP; donde $a$ es la ordenada al origen y $b$ es la pendiente. Además, se realizó un análisis de correlación de Pearson para valorar los grados de relación entre los distintos métodos directos e indirectos. Estos análisis se realizaron con una significancia de 0,05 en el programa STATISTICA 9.0 (Statsoft, 2015).

\section{Resultados}

\section{Comparación de los IAF obtenidos a través del método indirecto y el método directo}

Los valores del IAF obtenidos con el método directo en $E$. tereticornis variaron de $0,68 \mathrm{~m}^{2} \mathrm{~m}^{-2}$ a $2,67 \mathrm{~m}^{2} \mathrm{~m}^{-2}$; mientras que con el indirecto de LAI-2000 osciló de $0,88 \mathrm{~m}^{2} \mathrm{~m}^{-2}$ a 2,60 $\mathrm{m}^{2} \mathrm{~m}^{-2}$; para el FDH-app fue de $0,85 \mathrm{~m}^{2} \mathrm{~m}^{-2}$ a $2,54 \mathrm{~m}^{2} \mathrm{~m}^{-2} \mathrm{y}$ para el FDH-Lente varió de $0,80 \mathrm{~m}^{2} \mathrm{~m}^{-2}$ a $2,30 \mathrm{~m}^{2} \mathrm{~m}^{-2}$. Estas mediciones se realizaron a una temperatura de $(24,5 \pm 1,6)$ ${ }^{\circ} \mathrm{C}$, humedad relativa de $(75 \pm 10) \%$ y luminosidad de $(156789 \pm 24500)$ lux. En la figura 1 se presenta el IAF directo con respecto el IAF indirecto; de manera general, todos los modelos mostraron la tendencia que en IAF inferiores a $1,5 \mathrm{~m}^{2} \mathrm{~m}^{-2}$ se sobreestima el parámetro, mientras que para IAF superiores se subestimó. 


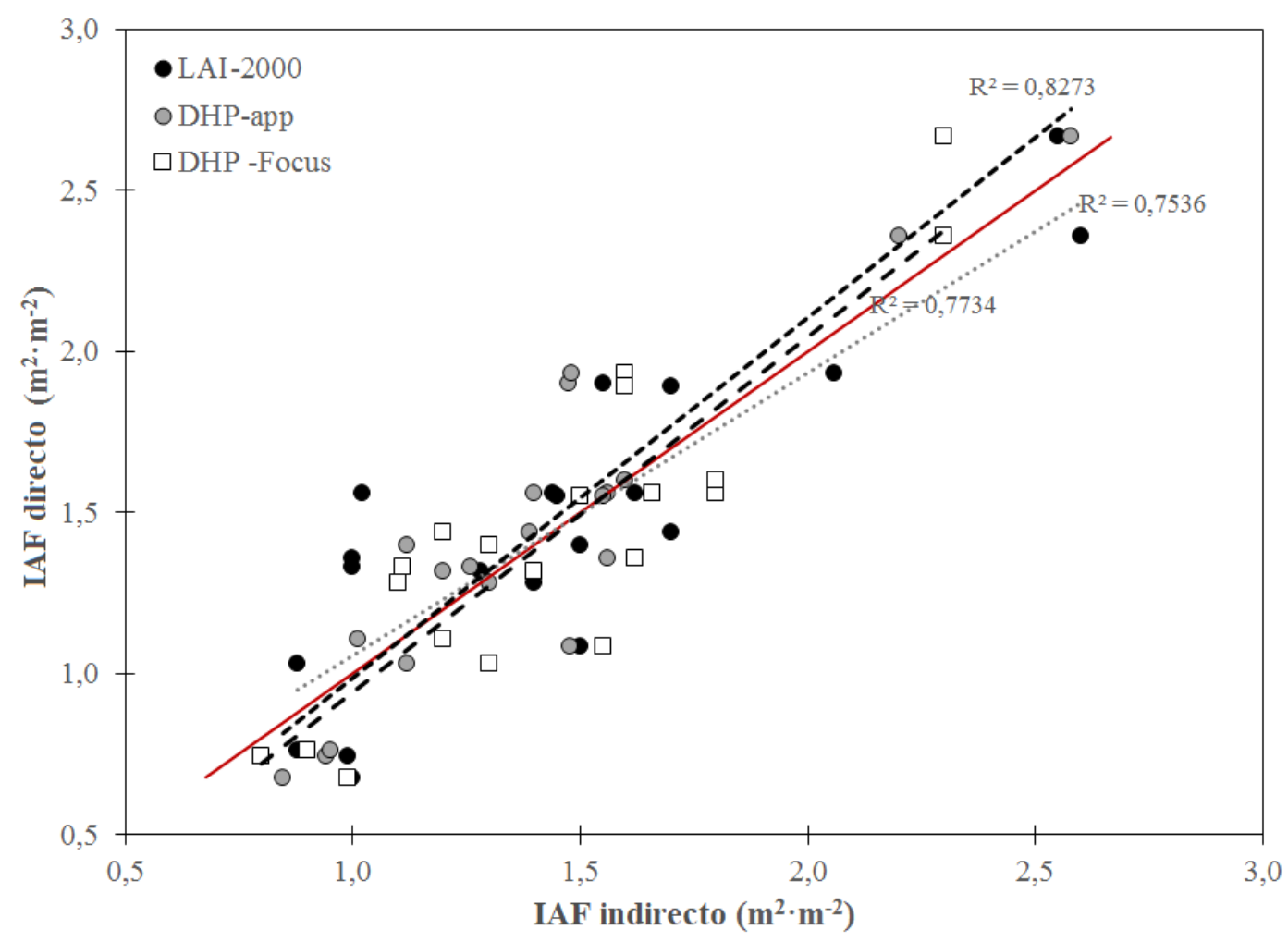

FIGURA 1. Valores del IAF indirecto obtenidos con el LAI-2000, FDH-app and FDH-Lente relacionados con de IAF directo de los individuos juveniles de E. tereticornis.

TABLA 1. Coeficientes de regresión del modelo IAF $=a \cdot I A P+b$ para determinar el IAF a partir del IAP en individuos juveniles de $E$. tereticornis, con una significancia de 0,05 .

\begin{tabular}{clcccc}
\hline IAF estimación & Parámetro & Estimado & Error estándar & Valor de t & Valor de $P$ \\
\hline \multirow{2}{*}{ LAI-2000 $\left(R^{2}=0,756\right)$} & Constante & 0,17 & 0,17 & 1,01 & 0,325 \\
& IAP & 0,88 & 0,11 & 7,81 & $<0,001$ \\
\hline \multirow{2}{*}{ FDH-app $\left(R^{2}=0,832\right)$} & Constante & $-0,13$ & 0,15 & $-0,78$ & 0,4423 \\
& IAP & 1,12 & 0,10 & 9,79 & $<0,001$ \\
\hline \multirow{2}{*}{ FDH-Lente $\left(R^{2}=0,773\right)$} & Constante & $-0,24$ & 0,22 & $-1,09$ & 0,2883 \\
& IAP & 1,17 & 0,15 & 7,91 & $<0,001$ \\
\hline
\end{tabular}

$\mathrm{Al}$ analizar los modelos obtenidos, se encontró que el mejor modelo fue el FDH-app con la ecuación IAF = $0,88 \cdot \mathrm{IAP}+0,17$, con una sobreestimación inferior a $15 \%$ en árboles de bajo IAF y una subestimación de 38\% en los individuos con los IAF mayores; además, el modelo mostró el mejor coeficiente de determinación $\left(\mathrm{R}^{2}\right)$ de 0,83 ; con una distribución normal de los residuos del parámetro IAP (P
$=0,325)$ y el error estándar de los parámetros de la ecuación inferiores a 0,16 (Tabla 1).

Con el instrumento LAI-2000 se obtuvo el modelo $\mathrm{IAF}=1,12 \cdot \mathrm{IAP}-0,13$ (Tabla 1), mostrando una tendencia de sobrestimar el IAF en $25 \%$ en los individuos pequeños y una subestimación inferior de $47 \%$ en los IAF mayores; igualmente este modelo obtuvo un valor de $\mathrm{R}^{2}$ de 0,76 con 
una distribución normal de los residuos del parámetro IAP $(\mathrm{P}=0,44)$, pero con errores estándar en sus parámetros de 0,17 a 0,11 (Tabla 1). En cuanto al modelo del FDH-Lente, con la ecuación IAF $=1,17 \cdot$ IAP-0,24, mostró subestimaciones inferiores a $20 \%$ en los IAF bajos y sobrestimaciones menores a $36 \%$ en los individuos de alto IAF, presentó un $\mathrm{R}^{2}$ del 77,3 con una distribución normal de los residuos del IAP $(\mathrm{P}=0,28)$, pero con errores estándar superiores a 0,15 siendo el mayor de los tres modelos indirectos (Tabla 1).

\section{Efectos de la nubosidad en la evaluación del IAF}

En la figura 2 se muestran los efectos de la nubosidad en el cálculo del IAF con los tres métodos indirectos de evaluación, se obtuvo un aumento de la subestimación del IAF en las condiciones de cielo parcialmente nublado. Con el LAI-2000 (Fig. 2a) el modelo en PN obtuvo la ecuación $\mathrm{IAF}=1,09 \cdot \mathrm{IAP}+0,28$ que tiende a subestimar todos los valores del IAF de $6 \%$ a $52 \%$ con respecto a TN y de $5 \%$ a $66 \%$ con respecto al IAF destructivo y con una leve disminución del $\mathrm{R}^{2}$ de 0,76 a 0,75. Por su parte, el FDHapp (Fig. 2b) con la ecuación IAF $=1,07 \cdot \mathrm{IAP}+0,06$, mantiene la tendencia a subestimar de $2 \%$ a $18 \%$ con respecto a TN y de $8 \%$ a $50 \%$ con el IAF directo, con una disminución de $\mathrm{R}^{2}$ de 0,83 (en TN) a 0,77. Finalmente, con el FDH-Lente con la ecuación IAF $=1,28 \cdot \mathrm{IAP}+0,07$, la subestimación varió de $5 \%$ a $45 \%$ con respecto a $\mathrm{CN}$ y de $8 \%$ a $60 \%$ con el método directo, pero se presentó un aumento de $\mathrm{R}^{2}$ de 0,76 (en TN) a 0,81 .

\section{Efectos de la velocidad del viento en la evaluación del IAF}

Con respecto al factor viento (Fig. 3), se encontró que conforme la velocidad del viento aumentó, el valor indirecto del IAF varió significativamente, generando sobrestimaciones con árboles con un IAF inferior a 1,7 $\mathrm{m}^{2} \mathrm{~m}^{-2}$ y subestimaciones con IAF mayores; con ello se da una disminución significativa en la precisión en los modelos. Con el VV2 (modelo IAF $=1,01 \cdot \mathrm{IAP}+0,21$ ), el factor viento presentó un comportamiento totalmente opuesto al VV1 (modelo con viento mínimo a nulo), sobrestimando el IAF hasta en $30 \%$ en individuos con un IAF con medición directa menores a $1 \mathrm{~m}^{2} \mathrm{~m}^{-2}$ y con valores superiores a este se dio una subestimación de $10 \%$ a 33\%, generando variaciones máximas de $60 \%$ con respecto a los IAF reales. El $\mathrm{R}^{2}$ del modelo fue de 0,14 (Tabla 2), con distribución normal de los residuos del IAP $(P=0,10)$ y errores estándar de sus parámetros superiores a 0,20. Un caso similar se dio con el VV3, que mantuvo el comportamiento de sobrestimar los menores IAF en $15 \%$ y un subestimado máximo de $40 \%$ en los IAF mayores, el valor del $\mathrm{R}^{2}$ del modelo fue de 0,37 (modelo IAF $=$ $0,46 \cdot \mathrm{IAP}+0,91)$, pero no se obtuvo una distribución normal de sus residuos $(\mathrm{P}=0,0043)$ y con errores estándar superiores a 0,14 , lo cual significa un modelo poco confiable. Finalmente, con respecto al VV4, la línea de tendencia es casi paralela al eje $\mathrm{X}$ (con la ecuación IAF = $0,17 \cdot \mathrm{IAP}+1,121)$, con un $\mathrm{R}^{2}$ de 0,04 además de errores estándar superiores a 0,20 y sin distribución normal de los residuos del IAP $(\mathrm{P}=0,041)$, que lo califica como un modelo ineficiente.

\section{DISCUSIÓN}

\section{FDH y LAl-2000 comparados con el método directo}

La tendencia a subestimar el IAF en árboles de medio y gran porte es similar a lo encontrado en estudios con Eucalyptus y con otras especies arbóreas (Breda, 2003). La tendencia de tener variaciones entre 30\% y 60\% es típica en los IAF, según mencionan Kucharik, Norman, y Gowerk (1998), con métodos destructivos en tres especies de eucalipto, Gower, Kucharik y Norman (1999) con Eucalyptus deglupta, estimado con equipos especializados LICOR, Küßner y Mosandl (2000), con cinco especies arbóreas de clima templado y Leblanc y Chen (2001), con seis especies de Eucalyptus evaluadas con cinco métodos ópticos. En el caso de mediciones con FDH, Mason et al., (2012) registraron variaciones de $10 \%$ a 60\% del IAF en Pinus radiata de 7 años de edad; por su parte Yan et al. (2019) con dos especies de Eucalyptus observaron variaciones inferiores a $45 \%$; en ambos 


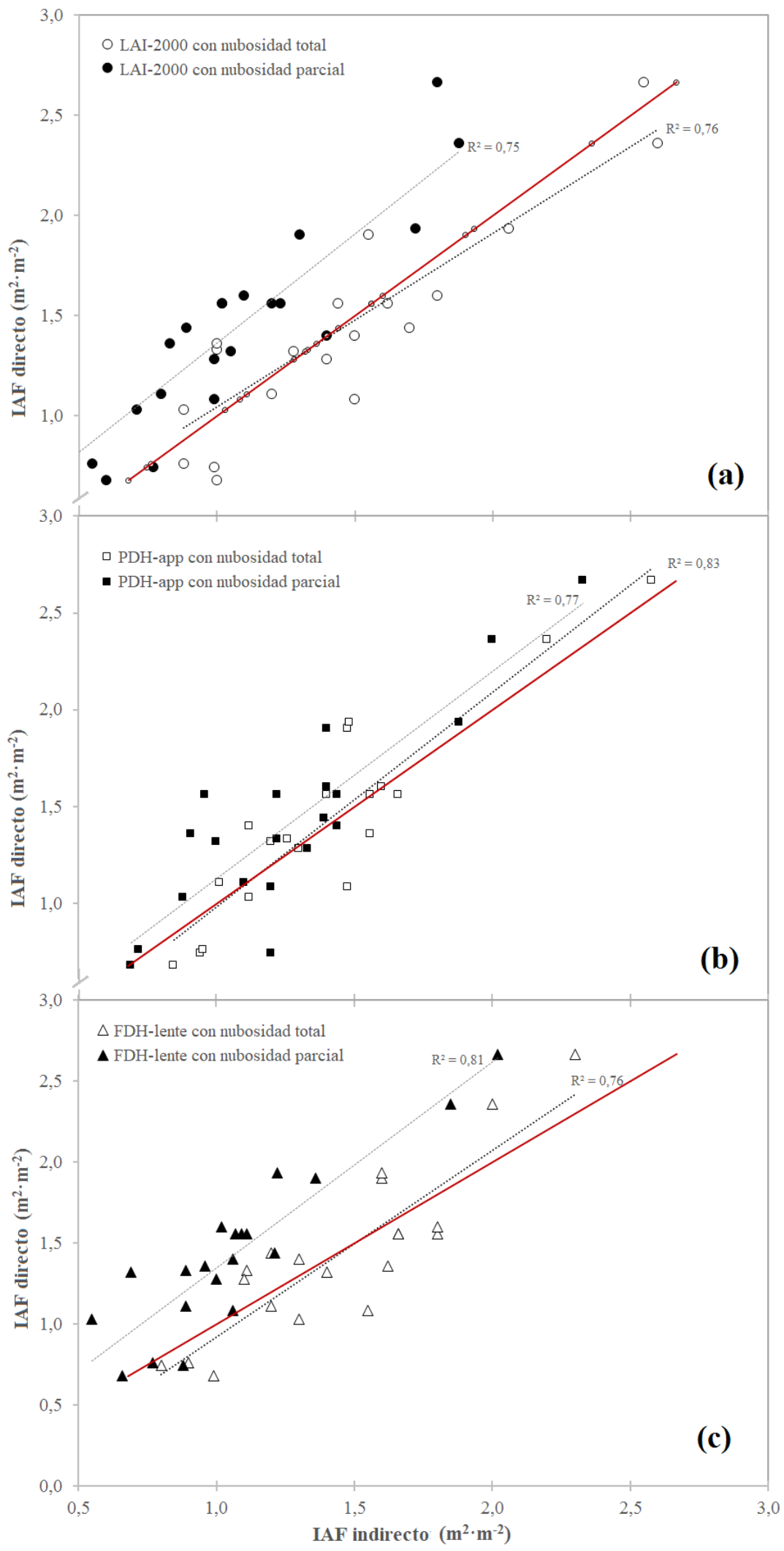

FIGURA 2. Valores del IAF indirecto obtenidos con el LAI-2000, FDH-app and FDH-Lente relacionados con de IAF directo en condiciones de clima total y parcialmente nublado en plantaciones juveniles de E. tereticornis 


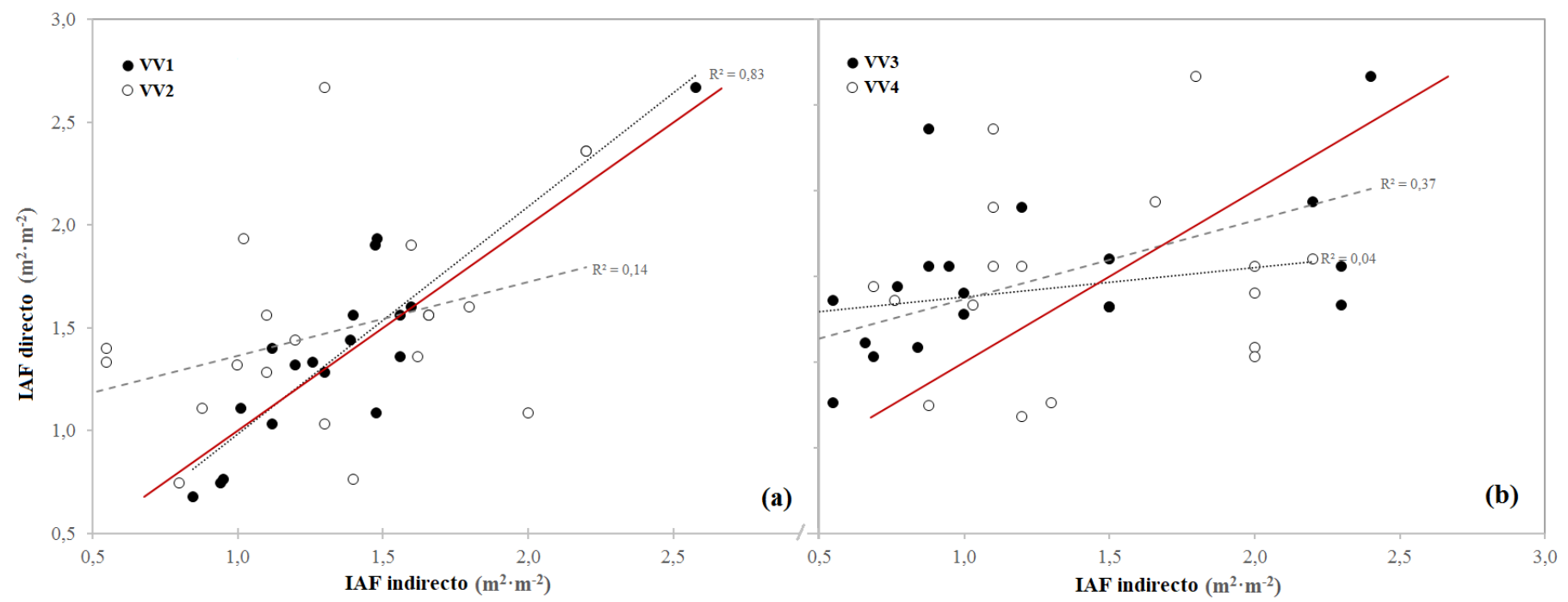

FIGURA 3. Valores del IAF indirecto obtenidos con FDH-app relacionado con de IAF directo bajo cuatro velocidades de viento en plantaciones juveniles de E. tereticornis

TABLA 2. Coeficientes de regresión de los de las ecuaciones de determinación del IAF a partir de IAP bajo el modelo Y $=$ aX $+\mathrm{b}$ obtenidos de las mediciones con FDH-app en cuatro intervalos de velocidad del viento en plantaciones juveniles de E. tereticornis

\begin{tabular}{llcccc}
\hline IAF estimación & Parámetro & Estimado & Error estándar & Valor de t & Valor de $P$ \\
\hline \multirow{2}{*}{ VV1 $\left(R^{2}=0,83\right)$} & Constante & $-1,13$ & 0,17 & $-0,73$ & 0,47 \\
& IAP & 1,11 & 0,12 & 9,44 & $<0,001$ \\
\hline \multirow{2}{*}{ VV2 $\left(R^{2}=0,14\right)$} & Constante & 1,01 & 0,27 & 3,69 & $<0,001$ \\
& IAP & 0,21 & 0,21 & 1,71 & 0,1051 \\
\hline \multirow{2}{*}{ VV3 $\left(R^{2}=0,37\right)$} & Constante & 0,91 & 0,19 & 4,89 & $<0,001$ \\
& IAP & 0,46 & 0,14 & 3,27 & 0,0043 \\
\hline \multirow{2}{*}{ VV4 $\left(R^{2}=0,04\right)$} & Constante & 1,21 & 0,29 & 4,18 & $<0,001$ \\
& IAP & 0,17 & 0,20 & 0,84 & 0,041 \\
\hline
\end{tabular}

casos se debió a los sesgos que generan los métodos ópticos. Dovey y du Toit (2005) y Confalonieri et al. (2013) mencionan que entre los aspectos que puede generar variaciones de los métodos ópticos está la distribución de las hojas y presencia de ramas. La FDH en el proceso de clasificación de pixeles de cobertura foliar en la gama de verdes puede variar significativamente por la luminosidad, generando en algunos casos que el sistema considere hojas como ramas debido al parecido de la cromática de pixeles. Algo similar se da con el borde de las hojas que tiende a confundirse con el cielo, lo cual influye en la pérdida del
IAF. Nguy-Robertson et al., (2014) destacan que la fotografía en modo automático tiende a aumentar el canal rojo y simplificar el verde, incidiendo con ello en una lectura de pixeles errónea en CAN-EYE. Por su parte Lui et al. (2013) destacaron que la luminosidad influye directamente en el IAF en sitios con altas densidades, generalmente se da el traslape de copas dificultando al investigador la selección correcta del cobertor en el sensor del LAI-2000 o en FDH el poder de recortar de manera óptima la imagen, evitando la eliminación de segmentos de ramas del individuo en estudio e incidiendo en la pérdida de información. 
En el caso de los árboles de menor porte se generó una sobrestimación del IAF; Dovey y du Toit (2005) con E. grandis de 24 meses de edad obtuvieron un comportamiento similar, con sobrestimaciones del IAF debidas a que lo individuos menores tienden a presentar gran cantidad de ramas y poca presencia de hojas que en el momento de hacer correcciones del IAP (en las cuales se elimina el efecto de ramas y fuste en el cálculo). Sobre esto en particular, los modelos algorítmicos confunden los pixeles de las hojas con los de las ramas debido al elevado grado de similitud de los colores, además de que la distribución de las hojas tiende a generar acumulaciones en algunos segmentos de la copa, lo cual implica un sesgo el cálculo.

Con respecto a la comparación de métodos (Tabla 3), la relación del FDH- app con el IAF directo $(0,91)$, que fue mayor en comparación al IAF-200 y FDH-Lente $(0,87)$, la deficiencia del cálculo de IAF del dispositivo móvil con lente de ojo de pez se debió a su poca estabilidad y a que no es totalmente compatible con toda cámara de dispositivo móvil. Confalonieri et al. (2013) destacan que el uso de aplicaciones para computadoras y teléfonos inteligentes para el cálculo del IAF genera buena precisión y confiabilidad en la medición debido a que se pueden definir y estandarizar variables como la curvatura de la imagen del ojo de pez, su relación con la luminosidad y la selección de un ISO, mientras con el LAI-2000, Chianucci, Macfarlane, Pisek, Cutini y Casa (2014) mencionan que leves variaciones en el ambiente influyen en el cálculo del IAF, específicamente en el ángulo de los rayos del sol que puede incidir en un aumento de la subestimación del IAF.

\section{Efectos del ambiente en el cálculo del IAF}

El efecto de subestimación del IAF en coberturas parcialmente nubladas se debe a un aumento en la radiación solar que incide en la reflexión de la luz por parte de la hoja conforme la nubosidad disminuye, lo cual genera pérdida de información en las imágenes, como es el caso del borde de la hoja que se puede confundir con pixeles del cielo; además, Chianucci et al. (2014) mencionan que en este tipo de condición se obtienen cambios en la sombra foliar y, en algunos casos, en las sombras con dos angulaciones distintas que generan aumento de errores de cálculo del IAF en el equipo LAI-2000, debido a que sus modelos algorítmicos no consideran estas variaciones.

Como se muestra en la tabla 4, el efecto del viento produce disminuciones significativas de correlación entre el IAF indirecto y el directo, hay variaciones entre $20 \%$ y $60 \%$ entre ambos. Estas variaciones son producto del movimiento que tiene la copa como respuesta al viento; si bien en la bibliografía disponible no se cuenta con otros estudios que analizan este fenómeno, se puede inferir que la disminución de las correlaciones (que llegan a ser de 30\% a 55\%) se debe a un cambio en la orientación y dirección de las hojas. Esto viola los principios de la Ley de Beer-Lambert y, con ello, genera variaciones que pueden llegar a ser aleatorias, incurriendo en una gran variabilidad de los datos. Mason et al. (2014) destacan que, conforme exista mayor homogeneidad y estabilidad de la copa, los valores ópticos del IAF serán mejores debido a que se cumplen los principios de Beer-Lambert. En este caso, al existir variabilidad por el efecto del viento, la precisión de mecanismos será mínima generando con ello errores y valores de correlación bajos.

TABLA 3. Coeficientes de correlación de Pearson de los valores de IAF obtenido con método directo e indirecto en árboles juveniles de E. tereticornis

\begin{tabular}{lcccc}
\hline & Directo & $\begin{array}{c}\text { Indirecto LAl- } \\
2000\end{array}$ & $\begin{array}{c}\text { Indirecto } \\
\text { FDH-app }\end{array}$ & $\begin{array}{c}\text { Indirecto FDH- } \\
\text { Lente }\end{array}$ \\
\hline Directo & 1 & & & \\
Indirecto LAI-2OOO & $0,87^{* *}$ & 1 & & \\
Indirecto FDH-app & $0,91^{* *}$ & $0,81^{* *}$ & 1 & \\
Indirecto FDH-Lente & $0,87^{* *}$ & $0,74^{* *}$ & $0,91^{* *}$ & 1 \\
\hline
\end{tabular}

ns, no hay significancia; *, significancia al 0,05 ; **, significancia al 0,01 
TABLA 4. Coeficientes de correlación de Pearson de los valores del IAF obtenidos por método directo e indirecto (FDH-app), en árboles juveniles de E. tereticornis

\begin{tabular}{lccccc}
\hline & Directo & SW1 & SW2 & SW3 & SW4 \\
\hline Directo & 1 & & & & \\
$V V 1$ & $0,91^{* *}$ & 1 & & & \\
$V V 2$ & $0,37^{\mathrm{ns}}$ & $0,49^{\mathrm{ns}}$ & 1 & 1 & \\
$V V 3$ & $0,61^{*}$ & $0,46^{*}$ & $-0,04^{\mathrm{ns}}$ & $0,41^{\mathrm{ns}}$ & 1 \\
$V V 4$ & $0,19^{\mathrm{ns}}$ & $0,20^{\mathrm{ns}}$ & $0,19^{\mathrm{ns}}$ & 0.06 \\
\hline
\end{tabular}

$n s$, no hay significancia; *, significancia al 0,05 ; **, significancia al 0,01

\section{Potencialidad y limitaciones de FDH de dispo- sitivos móviles para el cálculo del IAF}

Se demostró la viabilidad que tiene la utilización de FDH obtenidas con aplicaciones o lentes externos en dispositivos móviles y su ventaja al ser un sistema de evaluación rápido, con adaptabilidad a las condiciones del sitio, así como la flexibilidad de seleccionar y mejorar la calidad de las imágenes obtenidas, lo cual representa una ventaja sobre otras técnicas ópticas como el IAF -2000 (Chianucci et al., 2014); estos últimos son sistemas donde no se puede controlar en su totalidad la calidad de la información obtenida o en su defecto, eliminar elementos u objetos que generen distorsiones en las mediciones.

Confalonieri et al. (2013) y Piayda et al., (2015) mencionan que el IAF obtenido de fotografías hemisféricas no solo tiene mayor control en su proceso de medición, sino que también se puede adaptar mejor a distintas condiciones ambientales ya sea en áreas urbanas o con árboles aislados y rodeados por infraestructura que puede generar ruido en el cálculo. Bréda (2013) destaca su simplicidad de aplicación en distintos momentos del día, debido a que se puede manipular y adaptar la clasificación posterior de pixeles de acuerdo con la hora y condiciones climáticas, facilitando la estandarización de las categorías cromáticas de coberturas, como nubes y vegetación; con ello se puede, extender los periodos de evaluación del IAF, algo que es limitado con equipos como el LAI-2000, que depende de las mediciones que se realicen con ángulo de radiación solar inferiores a $45^{\circ}$ para simplificar errores por refracción de la luz.
Bréda (2003), Facci, Baroni, Boschetti y Gandol (2010) y Confalonieri et al. (2013) destacan que entre las principales limitaciones de este sistema de evaluación del IAF está la tendencia a subestimar sus valores dependiendo de la edad y arquitectura de la copa de cada especie evaluada; en el caso de Dovey y du Toit (2005), con E. grandis de 24 y 36 meses, encontraron variaciones significativas en el IAF de ambas especies, como producto de la dinámica de desarrollo de los individuos y en ella los árboles de 24 meses mostraron mayor subestimación que los de 36 meses, argumentando que son árboles con un ritmo de crecimiento mayor y con un nivel de competitividad elevado comparado a los de 36 meses. Por su parte Chiannucci et al. (2013) argumentan que en rodales de Pinus radiata las variaciones del IAF dependen de la densidad de siembra de los árboles, que se demuestra en los sitios con mayor densidad mediante un mayor traslape de las copas, lo cual dificulta diferenciar y delimitar claramente la copa de cada individuo, que a su vez provoca delimitaciones subjetivas que pueden incidir en subestimación del IAF, caso similar al presentado en estudios de Zhuo, Wang, Q; Li, M; Wang, R (2018), con plantaciones de cinco especies de eucalipto de dos a siete años de edad y Yan et al. (2019) con tres especies de eucalipto de cuatro años de edad. En ambos estudios se encontró que la forma reducida de la copa de los eucaliptos y la arqueadura que se genera por el viento y su tendencia a realizar ramificaciones con angulaciones inferiores a $40^{\circ}$, inducen a subestimar con vientos superiores a $10 \mathrm{~km} / \mathrm{h}$, debido al traslape que se da entre ramas y hojas. 


\section{CONCLUSIONES}

Se confirmó la viabilidad de utilizar FDH-app y FDHLente en el cálculo de IAF en E. tereticomis de 26 meses, obteniendo que el mejor método indirecto fue el FDH-app con el modelo IAF $=0,88 \cdot \mathrm{IAP}+0,17$ con coeficiente de correlación de 0,91 con respecto a los valores directos del IAF; seguido por el LAI-2000 y FDH-Lente que presentaron menor precisión y correlación $(0,87)$. Esto se debió a la facilidad de adaptación y uso las FDH.

Además, se determinó que los factores ambientales generan variaciones significativas del IAF: en el caso del factor nubosidad se obtuvo un aumento de la subestimación del IAF independientemente de la técnica indirecta empleada, con variaciones de hasta $60 \%$ con respecto al IAF real, pero manteniendo la tendencia del FDH-app que es el mejor sistema de evaluación. En cambio, se observó un aumento de la variabilidad de valores del IAF conforme la velocidad del viento aumentó, siendo las velocidades inferiores a $5 \mathrm{~km} / \mathrm{h}$ las óptimas para recolectar información, ya que las velocidades superiores inciden en variaciones de $10 \%$ a $65 \%$ en el IAF y disminuyen las correlaciones respecto a los valores reales hasta en $40 \%$.

Se recomienda que el uso de este tipo de metodologías se debe adaptar a la especie, condiciones del cultivo y objetivos de manejo que se busquen, ya que dichos aspectos influyen directamente en la adaptabilidad de la metodología propuesta, de forma tal que se debe realizar una prevaloración y validación.

\section{REFERENCIAS}

Bréda, N. (2003). Ground-based measurements of leaf area index: a review of methods, instruments and current controversies. Journal of Experimental Botany, 54(392), 2403-2417. doi: $10.1093 /$ jxb/erg263

Chen, J. M., Plummer, P. S., Rich, M., Gower, S. T., \& Norman, J. M. (1997) Leaf area index measurements. Journal Geophys Reseach, 102(29), 429-443.

Chianucci, F., \& Cutini, A. (2012). Digital hemispherical photography for estimating forest canopy properties: current controversies and opportunities. iForest, 5(6), 290-295. doi: 10.3832/ifor0775-005
Chianucci, F., \& Cutini, A. (2013). Estimation of canopy properties in deciduous forests with digital hemispherical and cover photography. Agricultural and Forest Meteorology, 168, 130-139. doi: $\underline{10.1016 / \text { j.agrformet.2012.09.002 }}$

Chianucci, F., Macfarlane, C., Pisek, J., Cutini, A., \& Casa, R. (2014). Estimation of foliage clumping from the LAI-2000 Plant Canopy Analyzer: effect of view caps. Trees, 29(6), 355-366. doi: $\underline{10.1007 / \mathrm{s} 00468-014-1115-\mathrm{x}}$

Confalonieri, R., Foi, M., Casa, R., Aquaro, S., Tona, E., Peterle, M., Boldini, A., Carli, G., Ferrari, A., Finotto, G., Guarneri, T., Manzoni, V., Movedi, E., Nisoli, A., Paleari, L., Radici, I., Suardi, M., Veronesi, D., Bregaglio, S., Cappelli, G., Chiodini, M.E., Dominoni, P., Francone, C., Frasso, N., Stella, T., \& Acutis, M., (2013). Development of an app for estimating leaf area index using a smartphone. Trueness and precision determination and comparison with other indirect methods. Computers and Electronics in Agriculture, 96(4), 1050-1062.

Demarez, V., Duthoit, S., Baret, F., Weiss, M., \& Dedieu, G. (2008). Estimation of leaf area and clumping indexes of crops with hemispherical photographs. Agricultural and Forest Meteorology, 148(1), 644-655. doi: 016/j.agrformet.2007.11.015

Dovey, S., \& du Toit, B. (2005). Calibration of LAI-2000 canopy analyser with leaf area index in a young eucalypt stand. Trees, 20(3), 273277. doi: $\underline{10.1007 / \mathrm{s} 00468-005-0038-\mathrm{y}}$

Facchi, A., Baroni, G., Boschetti, M., \& Gandol, C., (2010). Comparing optical and direct methods for leaf area index determination in a maize crop. Journal of Agriculture Engineer, 9(1), 33-40. doi: $\underline{10.2478 / \text { forj-2019-0011 }}$

Gower, S.T., Kucharik, C.J., \& Norman, J.M., (1999). Direct and indirect estimation of leafarea index, fAPAR, and net primary production of terrestrial ecosystems. Remote Sensors Environtal, 70(4), 29-51.

Leblanc, S.G., \& Chen, J.M., (2001). A practical scheme for correcting multiple scattering effects on optical IAF measurements. Agricultural and Forest Meteorology, 110(5), 125-139. doi: $\underline{10.1016 / \text { S0168-1923(01)00284-2 }}$

IMN(Instituto Meteorológico Nacional). 2018. Condiciones meteorológicas regionales. Recuperado de http:/wmw.imn.ac.cr

Jonckheere, I., Fleck, S., Nackaerts, K., Muys, B., Coppin, P., et al. (2004). Review of methods for in situ leaf area index determination: Part I. Theories, sensors and hemispherical photography. Agricultural and Forest Meteorology, 121, 19-35. doi: $\underline{10.1016 / \text { j.agrformet.2003.08.027 }}$

Kucharik, C.J., Norman, J.M., \& Gower, S.T. (1998). Measurements of branch area and adjusting leaf area index indirect measurements. Agricultural and Forest Meteorology, 91(5), 69-88. 
Küßner, R. \& Mosandl, R., (2000). Comparison of direct and indirect estimation of leaf area index in mature Norway spruce stands of eastern Germany. Canada Journal of Forest Research, 30(3), 440-447, doi: $\underline{10.1139 / \text { cjfr-30-3-440 }}$

Lang, A. (1986). Leaf-area and average leaf angle from transmission of direct sunlight. Australian Journal of Botanic, 34(3), 349-355.

LI-COR Biosciences. (2010). LAI-2000 Plant canopy analyser: instruction manual. LI-COR, Lincoln, Nebrasca.

Liu, Z., Jin, G, \& Qi, Y. (2012) Estimate of leaf area index in an oldgrowth mixed broadleaved-Korean Pine Forest in Northeastern China. PloS one, 7(6), e32155, doi: 10.1371/journal.pone.0032155.

Mason, E., Diepstraten, M., Pinjuv, G., Lasserre, J.P., (2012). Comparison of direct and indirect leaf area index measurements of Pinus radiata D. Don. Agricultural and Forest Meteorology, 166(5), 113-119, doi: 10.1007/s10342-019-01221-2.

Mougin, E., Demarez, V., Diawara, M., Hiernaux, P., Soumaguel, N., \& Berg, A. (2014). Estimation of IAF, fAPAR and fCover of Sahel rangelands (Gourma, Mali). Agricultural and Forest Meteorology, 198199(2), 155-167, doi: 10.1016/j.agrformet.2014.08.006.

Nackaerts, K., Coppin, P., Muys, B., \& Hermy, M., (2000). Sampling methodology for IAF measurements with LAI-2000 in small forest stands. Agricultural and Forest Meteorology, 101(2): 247-250, doi: 10.1016/S0168-1923(00)00090-3.

Nguy-Robertson, A., Peng, Y., Gitelson, A., Arkebauer, T., Pimstein, A., Herrmann, I., Karnieli, A., Rundquist, D. \& Bonfil, D. (2014). Estimating green IAF in four crops: Potential of determining optimal spectral bands for a universal algorithm. Agricultural and Forest Meteorology, 192-193(2), 140-148.

Piayda, A., Dubbert, M., Werner, C., Correia, A., Pereira, J., \& Cuntz, M. (2015). Influence of woody tissue and leaf clumping on vertically resolved leaf area index and angular gap probability estimates. Forest Ecology and Management, 340, 103-113, doi: 10.1016/j.foreco.2014.12.026

Pisek, J., Ryu, Y., \& Alikas, K., (2011). Estimating leaf inclination and Gfunction from leveled digital camera photography in broadleaf canopies. Trees, 25, 919-924. doi: 10.1007/s00468-011-0566-6

Poblete-Echeverría, C., Fuentes, S., Ortega-Farias, S., Gonzalez-Talice, J., \& Yuri, J. (2015). Digital Cover Photography for Estimating Leaf Area Index (IAF) in Apple Trees Using a Variable Light Extinction Coefficient. Sensors, 15(4), 1-8. doi: $\underline{10.3390 / \mathrm{s} 150202860}$

Statsoft. (2015). STATISTICA, version 9.0. London: Statsoft. Recuperado de http://www.statsoft.com
Valverde, J. C., Guevara-Bonilla, M., Arias, D., Briceño, E. y Esquivel, E. (2017). Efectos de las actividades de labranza en el índice de área foliar en una plantación de Tectona grandis en la zona norte de Costa Rica. Madera y Bosques, 23(2), 7-19. doi: $10.21829 /$ myb.2017.232498

Vojtech, E., Turnbull, L.A., \& Hector, A. (2007). Differences in light interception in grass monocultures predict short-term competitive outcomes under productive conditions. PloS one, 2(1): e499. doi: $\underline{10.1371 / \text { journal.pone.0000499 }}$

Vyas, D., Mehta, N., Dinakaran, A., \& Krishnayya, H. (2010). Allometric equations for estimating leaf area index (IAF) of two important tropical species (Tectona grandis and Dendrocalamus strictus). Journal of Forestry Research, 21, 197-200. doi: 10.1007/s11676-010-0032-0

Weiss, M., Baret, F., Smith, G.J., Jonckheere, I., \& Coppin, P., (2004). Review of methodsfor in situ leaf area index (IAF) determination. Part II. Estimation of IAF, errorsand sampling. Agricultural and Forest Meteorology, 121(5), 37-53.

Yan, G., Hu, R., Luo, J., Weiss, M., Jiang, H., Mu, M., Donghui, X., \& Zhang, W. (2019). Review of indirect optical measurements of leaf area index: Recent advances, challenges, and perspectives. Agricultural and Forest Meteorology, 265(4), 390-411. doi: $\underline{10.1016 / \text { j.agrformet.2018.11.033 }}$

Zhou, L., Wang, Q., Li, Y., Liu, M., Wang, R. (2018). Geen roof simulation with seasonally variable leaf area index Energy and building, 174(4), 156-167, doi: $10.1007 /$ s12273-018-0488-y

Zou, X., Mõttus, M., Tammeorg, P., Torres, C., Takala, T., Pisek, J., Mäkelä, P., Stoddard, F., Pellikka, P. (2014). Photographic measurement of leaf angles in field crops. Agricultural and Forest Meteorology, 184, 137-146. doi: 10.1016/j.agrformet.2013.09.010

\section{Manuscrito recibido el 15 de abril de 2019}

Aceptado el 16 de septiembre de 2019

Publicado el 24 de abril de 2020

\section{Este documento se debe citar como:}

Valverde, J. C. \& Arias، A. (2020). Evaluación del índice de área foliar con método indirecto y directo en distintas condiciones ambientales en plantaciones dendroenergéticas de Eucalyptus tereticornis Sm. Madera y Bosques, 26(2), e2621953. doi: 10.21829/myb.2020.2621953

Madera y Bosques por Instituto de Ecología, A.C. se distribuye bajo una Licencia Creative Commons Atribución-NoComercialCompartirlgual 4.0 Internacional. 